\title{
INCIDÊNCIA DE TUBERCULOSE NAS PENITENCIÁRIAS DA PARAÍBA DE 2007 A 2014
}

\section{INCIDENCE OF TUBERCULOSIS IN PENITENTIARIES OF PARAIBA FROM 2007 TO 2014}

Recebido em: 26/07/2018.

Aceito em: 29/10/2018.
Larissa Edilza de Lima ${ }^{1}$ Alynne Pires Fonsêca ${ }^{2}$ Polyana Maria Cruz Collaço ${ }^{3}$ Vivianne Almeida da Nóbrega ${ }^{4}$ Rayanne Rodrigues Santos ${ }^{5}$

\section{RESUMO}

O aprisionamento de seres humanos é um forte fator de risco para a infecção pela tuberculose, sendo a incidência de tuberculose nos presídios 11 a 81 vezes maior que a da população em geral, o que se explica por uma combinação de fatores como diagnóstico tardio, tratamento inadequado, superpopulação, ventilação precária e alta taxa de transferência entre penitenciárias. Desse modo, objetiva-se com esse estudo avaliar a incidência de tuberculose nos presídios da Paraíba nos anos de 2007 a 2014 e observar se os casos de tuberculose nos presídios do estado seguem a incidência nacional. Vale destacar que a população privada de liberdade tem 28 vezes maior chances de ter tuberculose, onde o atraso no diagnóstico relacionase à naturalização da desassistência ao sujeito preso o que auxilia na perduração e permanência da alta incidência da doença. Para realizar esse estudo, foram coletados dados do Departamento de Informática do Sistema Único de Saúde (DATASUS) e do Levantamento Nacional de Informações Penitenciárias (INFOPEN). Como resultados, observou-se a taxa de ocupação de $140 \%$, o que reflete na saúde dos presidiários. Além disso, em todo o estado tem-se os presídios como $3^{\circ}$ local com maior incidência de tuberculose, sendo 93\% dos casos em homens, 79,3\% dos casos na faixa etária de 2039 anos, predominantemente pardos e com nível de escolaridade entre a $1^{\text {a }}$ e a $4^{\mathrm{a}}$ série

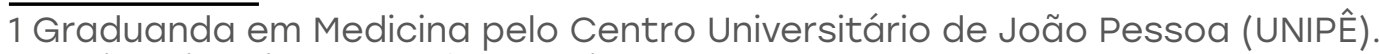

E-mail: larissalima_2013@hotmail.com

2 Graduanda em Medicina pelo Centro Universitário de João Pessoa (UNIPÊ).

E-mail: alynnepires7@hotmail.com

3 Graduanda em Medicina pelo Centro Universitário de João Pessoa (UNIPÊ).

E-mail: polyanacolaco@gmail.com

4 Graduanda em Medicina pelo Centro Universitário de João Pessoa (UNIPÊ).

E-mail: vivianne8nobrega@gmail.com

5 Graduanda em Medicina pelo Centro Universitário de João Pessoa (UNIPÊ).

E-mail: rayannersantos@gmail.com 
do ensino fundamental incompleto. Desse modo, percebe-se que é preciso desenvolver estratégias para diminuir tal incidência, melhorando a assistência à saúde e vida, com diagnóstico precoce e tratamento adequado.

Palavras-chave: Tuberculose. Prisões. Saúde. Epidemiologia.

\section{ABSTRACT}

The imprisonment of human beings is a strong risk factor for tuberculosis infection, with the incidence of tuberculosis in prisons 11 to 81 times higher than that of the general population, which is explained by a combination of factors such as late diagnosis, inadequate treatment, overpopulation, poor ventilation and high transfer rates between penitentiaries. Thus, the objective of this study is to evaluate the incidence of tuberculosis in Paraíba prisons in the years 2007 to 2014 and to observe whether the cases of tuberculosis in prisons in the state follow the national incidence. It is worth mentioning that the population deprived of freedom is 28 times more likely to have tuberculosis, where the delay in diagnosis is related to the naturalization of the detainee's lack of attendance, which assists in the survival and permanence of the high incidence of the disease. To carry out this study, data were collected from the Department of Informatics of the National Health System (DATASUS) and the National Survey of Penitentiary Information (INFOPEN). As a result, the occupancy rate of $140 \%$ was observed, which reflects on the prisoners' health. In addition, prisons as a third place with the highest incidence of tuberculosis, $93 \%$ of cases in men, $79.3 \%$ of cases in the age group of 20-39 years, predominantly brown race and between the 1 st and 4th grades of incomplete primary education. In this way, it is perceived that strategies need to be developed to reduce this incidence, improving health and life assistance, with early diagnosis and appropriate treatment.

Keywords: Tuberculosis. Prisons. Health. Epidemiology.

\section{INTRODUÇÃO}

A tuberculose (TB), sendo um agravo em saúde pública com grande magnitude, transcendência e vulnerabilidade, deve permanecer como assunto prioritário para profissionais de saúde e para a população em geral (BRASIL, 2011). Além disso, seu controle deve ser inserido em um panorama em que haja o uso organizado e contínuo das opções diagnósticas e terapêuticas disponíveis (SILVA, 2012).

Nesse contexto, estudos têm sido realizados buscando-se minimizar as repercussões negativas da doença e atender às necessidades das populações mais vulneráveis, como a carcerária, por exemplo. Ademais, é preciso que os gestores públicos, 
profissionais de saúde e a sociedade como um todo desenvolvam uma estratégia ativa para lidar com tal agravo, enfatizando o quadro epidemiológico para, a partir dele, buscar o paciente infectado pelo bacilo e seus contatos (SILVA, 2012).

No ambiente prisional, considerando-se os dados globais, a notificação da tuberculose é de 11 a 81 vezes maior que a da população em geral, o que se explica por uma combinação de fatores como diagnóstico tardio, tratamento inadequado, superpopulação, ventilação precária e alta taxa de transferência entre penitenciárias. Tal conjuntura é ainda mais complicada pelo advento e incremento de populações bacilíferas resistentes às drogas disponíveis (OMS, 2014).

No Brasil, dados de 2017 do Programa Nacional de Controle da Tuberculose mostram que enquanto a média da incidência da tuberculose na população total é de 33,5 por 100 mil habitantes, a da população privada de liberdade é de 1036,3 por 100 mil pessoas presas. Na região Nordeste, cinco estados (Sergipe, Rio Grande do Norte, Pernambuco, Maranhão e Bahia) apresentam números superiores aos da média nacional para o ambiente prisional (BRASIL, 2018). Nesse cenário, estudo publicado em 2015 apontou o cárcere como poderoso fator de risco para a infecção pela tuberculose, sendo a epidemia prisional desta doença relacionada com a da população em geral (SACCHI et al., 2015).

De acordo com a OMS (2014), em indivíduos em privação de liberdade com suspeita de tuberculose pulmonar deve ser feita a coleta de duas amostras de escarro para realização de baciloscopia direta. Outros métodos diagnósticos incluem a cultura do bacilo - importante para indivíduos com HIV e para a identificação da resistência bacteriana às drogas através do teste de sensibilidade - e a radiografia de tórax, útil nos casos de pacientes com suspeita de tuberculose, mas com resultado negativo na microscopia.

Quanto ao tratamento para as populações privadas de liberdade, este segue os mesmos esquemas para a população livre, não devendo ser supervisionado por profissionais da segurança e sim por trabalhadores da área da saúde. Além disso, é importante que haja um sistema de informação eficaz entre as unidades prisionais e notificação adequada dos casos (BRASIL, 2011).

Logo, é imperativo que, em populações especiais - como a de indivíduos em privação de liberdade -, haja uma busca de indivíduos com tuberculose, visando a um diagnóstico precoce, minimização da transmissão do bacilo e seguimento satisfatório do tratamento, com diminuição dos índices de abandono e incremento dos de cura (SILVA, 2012). 


\section{OBJETIVOS}

\section{OBJETIVO GERAL}

Pretende-se avaliar a incidência de tuberculose nas penitenciárias da Paraíba de 2007 a 2014.

\section{OBJETIVOS ESPECÍFICOS}

- Correlacionar os dados da população prisional dos dados com os casos de tuberculose;

- Avaliar as causas da incidência de tuberculose nos presídios;

- Avaliar as características do aprisionamento brasileiro, como por exemplo a superlotação, e observar como isso afeta na predisposição à doença;

\section{REVISÃO BIBLIOGRÁFICA}

A tuberculose é uma doença de caráter infeccioso e potencialmente transmissível. Esta possui como agente etiológico o Mycobacterium tuberculosis, que pode ter sua afecção pulmonar ou até mesmo, extrapulmonar (BRASIL, 2017). A transmissão dar-se por via aérea através de gotículas de uma pessoa infectada, de tal modo, sendo bastante encontrada em ambientes como presídios já que, muitas vezes, nestes ambientes existem alguns fatores de risco que contribuem para o desenvolvimento da tuberculose, incluindo pouca ventilação, superlotação, condições sanitárias adversas, baixo nível socioeconômico, disseminação do HIV e drogadição (ANDRZEY VSKI; LIMBERGER, 2013).

Segundo Walter Tavares (2015), as taxas de morte ocasionadas por tuberculose, como também a prevalência e a incidência são afetadas tanto pelas condições socioeconômicas, quanto pela detecção de casos e tratamento. E ainda de acordo com Andrzeyvski e Limberger (2013), demonstrou-se que a TB acomete as pessoas na fase mais produtiva da vida, do sexo masculino, possuindo baixa escolaridade e poder socioeconômico desfavorecido.

Aliado a isso, denotou-se que há relação diretamente proporcional entre a infecção e o tempo de encarceramento. Na medida em que o tempo de prisão aumenta, progressivamente, aumenta-se o número de presos infectados pela TB. Assim como há diferença de incidência de infecção entre detentos primários e reincidentes, nestes, a taxa de incidência apresenta-se maior. Ou seja, é possível identificar como um fator de risco de infecção por $\mathrm{TB}$, o tempo de permanência do indivíduo na prisão e o retorno à prisão. Quanto ao regime de cumprimento de pena, o maior número de infectados encontrava-se no regime fechado (MACHADO et al., 2016).

É sabido que praticamente todos os seres humanos estão vulneráveis à infecção pelo Mycobacterium tuberculosis. No entanto, há populações especificas que apresentam 
um maior risco ao adoecimento e acometimento da tuberculose. De acordo com Sistema de Controle de Pacientes com Tuberculose, por conta das suas condições sanitárias e de saúde, a população privada de liberdade tem 28 vezes maior chances de ter tuberculose, já a população indígena possui 3 vezes mais chance de desenvolver a doença (BRASIL, 2017).

Ainda de acordo com o Ministério da Saúde (2016), a população, em geral, tem como incidência de tuberculose 33 casos por 100 mil habitantes, já na população carcerária há mais de 900 casos para 100 mil habitantes. Tratando-se assim, de um caso nítido de emergência de saúde pública. Ademais, segundo Valença et al. (2016), sugerese que a tuberculose em pessoas privadas de liberdade está associada a características individuais e a condição de vida antes mesmo do encarceramento, além de fatores ambientais relacionados à reclusão.

Estudos sobre a saúde da população encarcerada no Brasil e nos países em desenvolvimento evidenciam os mesmos problemas que em outros continentes, inclusive o europeu, com a crescente taxa de ocupação prisional, sem a concomitante adequação de estrutura física e de recursos humanos, somada às condições precárias de higiene, ventilação e iluminação solar nas celas. Estes fatores resultam na ocorrência da TB em presídios, sendo um preocupante problema de saúde pública, devido tamanha magnitude (ALVES et al., 2012 apud ANDRZEYVSKI; LIMBERGER, 2013).

De acordo com as metas propostas pelo Programa Nacional de Controle da Tuberculose (PNCT), deveria existir uma detecção anual de pelo menos 70\% dos casos de tuberculose e a cura de pelo menos $85 \%$ dos pacientes diagnosticados com tuberculose. Para isso, recomenda-se a detecção com base na demanda espontânea dos presidiários, busca ativa, rever indicação de cultura e teste de sensibilidade, exames para avaliar se há coinfecção com o vírus HIV, supervisão do tratamento e informação e conscientização das pessoas privadas de liberdade e guardas, além de melhoria das condições ambientais (BRASIL, 2011). Apesar das altas taxas de prevalência, observou-se que, de forma geral, as equipes de saúde seguem a maior parte das normas e recomendações, com maior envolvimento dos profissionais de enfermagem (MACHADO et al., 2016).

Além disso, é importante destacar que a desinformação quanto à infecção de TB é tão alta quanto sua taxa de infecção nos detentos, conforme os dados levantados (MACHADO et al., 2016). Essa desinformação colabora com os atrasos no diagnóstico da doença sendo que a detecção precoce dos casos de tuberculose (TB) representa uma atividade essencial para o controle da doença (PONCE et al., 2016).

Segundo Souza et al. (2012), o atraso no diagnóstico da tuberculose relacionase à naturalização da desassistência ao sujeito preso, à interpretação do presídio como um lugar de morte e sofrimentos e à privação do direito à saúde para detentos em decorrência de sua posição nas relações assimétricas de poder e efeitos ideológicos. Tais fatos evidenciam a precária situação da prevenção da TB, com a baixa qualidade no atendimento dos infectados (MACHADO et al., 2016). 


\section{METODOLOGIA}

Trata-se de um estudo epidemiológico descritivo realizado por meio da análise de dados do Departamento de Informática do Sistema Único de Saúde (DATASUS) e do Levantamento Nacional de Informações Penitenciárias (Infopen). Foram obtidos no Infopen os dados relacionados à população carcerária brasileira, dando enfoque ao número de presos no estado da Paraíba, a taxa de presos a cada 10.000 habitantes e o número de vagas disponibilizadas pelo sistema carcerário do estado. A amostra estudada no DATASUS foi constituída por todos os casos novos de tuberculose notificados nas penitenciárias do estado da Paraíba no período de 2007 a 2014. Analisaram-se as variáveis: casos novos, tipo de entrada, sexo, raça, faixa etária, nível de escolaridade, forma de tuberculose e tratamento. Sendo realizado posteriormente a porcentagem de cada uma das variáveis a fim de se obter o perfil epidemiológico da população que está sendo afetada por essa patologia.

\section{RESULTADOS E DISCUSSÃO}

De acordo com os dados de 2014 da Infopen, o Brasil encontra-se em quarto lugar no ranking de países com maior população prisional do mundo, ficando atrás apenas dos Estados Unidos, da China e da Rússia. A população carcerária brasileira é constituída por 622.202 indivíduos e esses números têm aumentado rapidamente fazendo com que haja um déficit de vagas e, consequentemente, uma superlotação dos presídios.

Em diferentes estados, estudos comparando a incidência da TB nas populações privadas de liberdade e na população geral coincidem ao mostrar maiores taxas entre as primeiras. Na Paraíba, entre 2000 e 2005, a incidência foi 21 vezes maior (FORMIGA; LIMA, 2011; VITTI JR., 2013). Entre os anos de 2000 a 2005, houve um total de 165 casos de TB dos 7.272 presidiários do estado da Paraíba, sendo 23 casos no ano de 2000, 21 casos em 2001, 17 casos em 2002, 27 casos em 2003, 36 casos em 2004 e 41 casos em 2005 (FORMIGA; LIMA, 2011; SOARES et al., 2015), o que denota uma certa estabilidade no número de casos.

No estado da Paraíba são 10.450 presos, o que corresponde a uma taxa de 26,47 indivíduos a cada 10.000 habitantes. Entretanto, a Paraíba apresenta um total de 7.488 vagas no sistema prisional, ou seja, as estatísticas evidenciam a superlotação que está ocorrendo nos presídios paraibanos, os quais apresentam uma taxa de ocupação de 140\% que está sendo refletida na saúde dos indivíduos que estão residindo nesses locais.

No Gráfico 1, têm-se os dados referentes ao número total de casos de tuberculose no estado da Paraíba no período de 2007 a 2014, onde foram notificados 8.435 casos novos de TB. Ao realizar uma análise dos casos ao decorrer de cada ano, nota-se que o número de casos apresentou períodos de crescimento e declínio, porém não foram valores consideráveis de mudança, o que denota estabilidade do número de 
casos com o decorrer dos anos, sendo 992 casos em 2007, 1.053 casos em 2008, 1.037 casos em 2009, 1.016 casos em 2010, 1.076 casos em 2011, 1.131 casos em 2012, 1.115 em 2013 e 1.015 em 2014.

Gráfico 1 - Incidência de Tuberculose no Estado da Paraíba, no período de 2007 a 2014

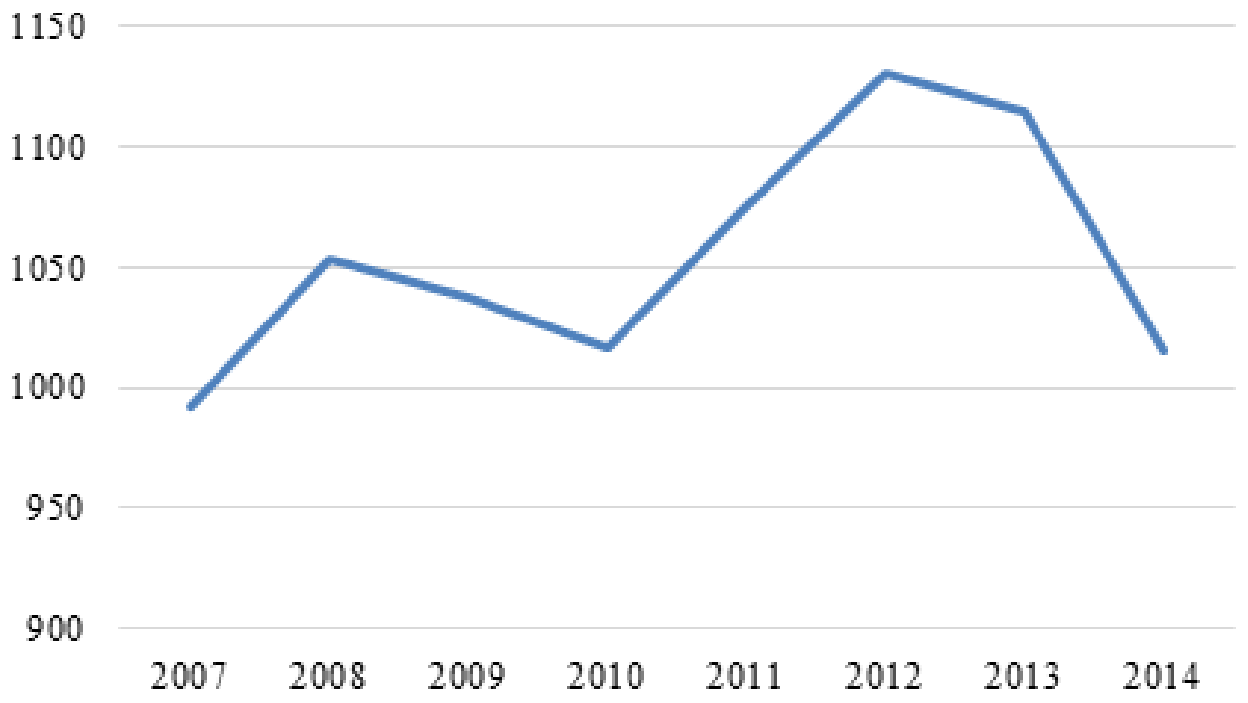

Fonte: Dados do Sistema de Informação de Agravos de Notificação - Ministério da Saúde (2018)

Na Tabela 1, encontram-se os dados referentes ao tipo de institucionalização da incidência de tuberculose, observando que de todos os casos que ocorreram na Paraíba, destacam-se 606 casos procedentes dos presídios do estado, evidenciando os presídios como a terceira principal institucionalização de novos casos de tuberculose. Tabela 1 - Casos de Tuberculose no Estado da Paraíba, no período de 2007 a 2014,
de acordo com o Institucionalizado

\begin{tabular}{|l|l|}
\hline Institucionalizado & Casos confirmados \\
\hline Ignorado/Branco & 959 \\
\hline Não & 6.349 \\
\hline Presídio & 606 \\
\hline Asilo & 9 \\
\hline Orfanato & 16 \\
\hline Hospital psiquiátrico & 11 \\
\hline Outros & 485 \\
\hline Total & 8.435 \\
\hline
\end{tabular}

Fonte: Dados do Sistema de Informação de Agravos de Notificação - Ministério da Saúde (2018)

Nesse contexto, ressalta-se que a maioria dos casos de tuberculose nos presídios brasileiros se dá por meio de infecções novas adquiridas no próprio espaço prisional e não mediante a reativação de processos infecciosos prévios. Esse fato é resultado de um somatório de falhas estruturais, como má ventilação e iluminação nas prisões, número 
de detentos que ultrapassa a capacidade carcerária, deficiência do sistema de saúde em tal ambiente, além de aspectos sociais, relacionados à marginalização do indivíduo privado de liberdade pela população em geral e, muitas vezes, pelo Estado (LAROUZÉ et al., 2015).

Quanto à distribuição dos casos notificados, de acordo com o sexo, houve um predomínio do sexo masculino, foram 563 casos em homens e 43 em mulheres, observou-se que temos uma relação de aproximadamente 13 homens com TB para cada 1 mulher que apresenta a doença. Tal fato pode ser explicado pela nítida predominância masculina no ambiente prisional; dados do Banco Nacional de Monitoramento de Prisões (Conselho Nacional de Justiça) evidenciam que cerca de 95,5\% da população carcerária do estado da Paraíba é composta por homens, semelhante à porcentagem nacional, que é de $95,1 \%$.

Em relação a faixa etária (Gráfico 2), temos que 79,3\% dos casos de tuberculose notificados nos presídios ocorrem em indivíduos que se encontram entre 20-39 anos. Essas pessoas são predominantemente pardas, representando 71,1\% (Gráfico 3) e 30,4\% possuem escolaridade da $1^{\mathrm{a}}$ a $4^{\mathrm{a}}$ série do ensino fundamental incompleta (Gráfico 4).

Segundo o estudo de Mariana Soares Valença et al. (2016), observou-se que o predomínio de tuberculose é mais prevalente entre a faixa etária de 25 a 34 anos, correspondendo cerca de $63 \%$ dos presidiários. De tal modo, assemelha-se ao estudo realizado visto que, constatou que a maior prevalência se encontra na faixa etária 2039 anos. Além disso, analisou-se uma deficiência em estudos relacionando a cor dos presidiários e tuberculose, sendo necessários mais estudos para melhor compreensão e elucidação.

Além disso, de um modo geral, dentre as variáveis sociodemográficas de nível de escolaridade, o analfabetismo e o ensino fundamental incompleto (entre a $1^{\mathrm{a}}$ e $4^{\mathrm{a}}$ série ou $5^{\mathrm{a}}$ e $8^{\mathrm{a}}$ série) retêm as maiores porcentagens de indivíduos que abandonam o tratamento. Assim como os pacientes que pertencem às classes $\mathrm{C} 1, \mathrm{C} 2, \mathrm{D}$ e $\mathrm{E}$, de acordo com os critérios da Associação Brasileira de Estudos Populacionais (ABEP), também obtém as maiores porcentagens. Portanto, a menor informação sobre a doença, sendo consequência principal do baixo nível educacional, e correlação com a pobreza são considerados fatores preditivos do abandono do tratamento da tuberculose (SÁ et al., 2017). 
Gráfico 2 - Casos confirmados de TB nos presídios paraibanos por Faixa Etária

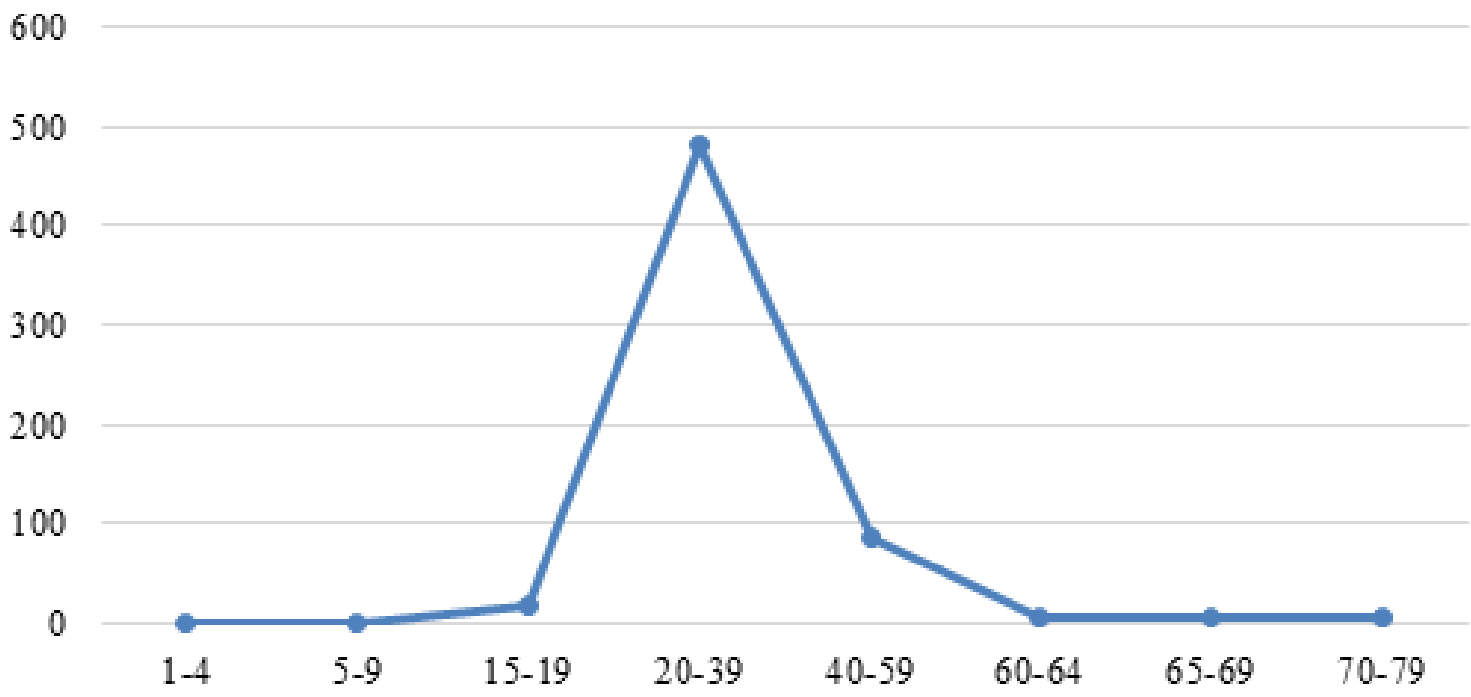

Fonte: Dados do Sistema de Informação de Agravos de Notificação - Ministério da Saúde (2018)

Gráfico 3 - Casos confirmados de TB nos presídios paraibanos por Raça

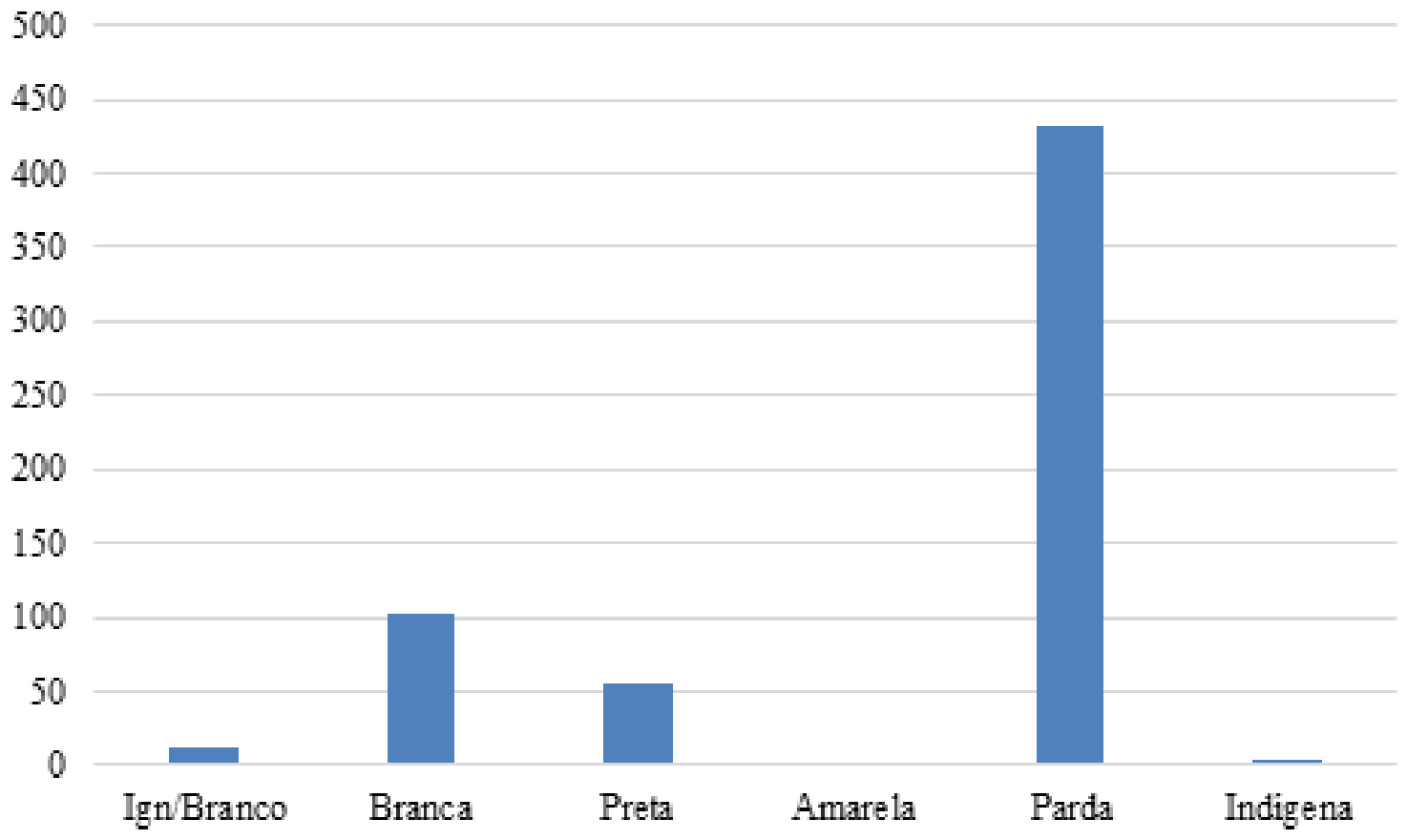

Fonte: Dados do Sistema de Informação de Agravos de Notificação - Ministério da Saúde (2018) 
Gráfico 4 - Casos confirmados de TB nos presídios paraibanos por Nível de Escolaridade

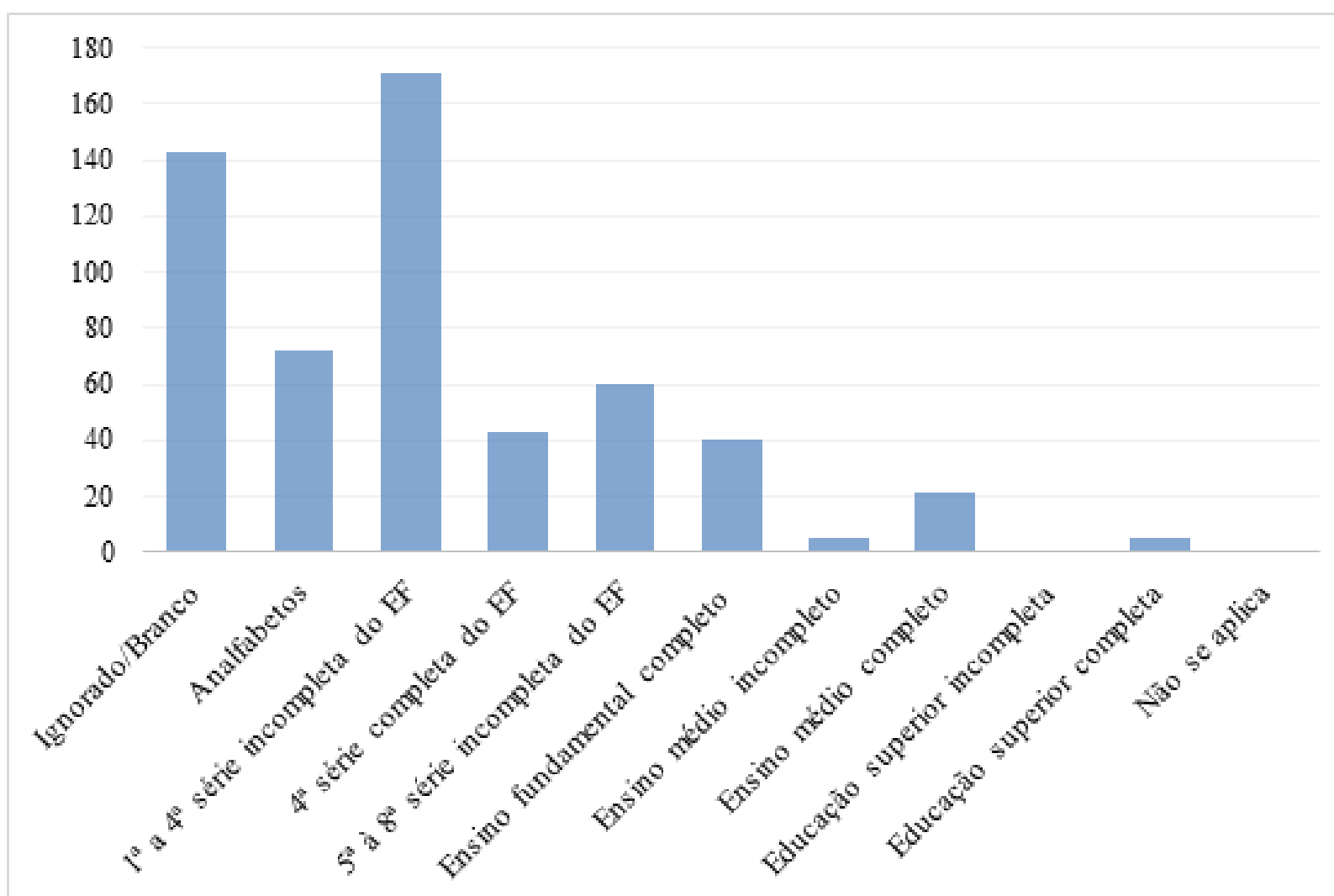

Fonte: Dados do Sistema de Informação de Agravos de Notificação - Ministério da Saúde (2018)

Entre as formas de tuberculose, a forma pulmonar destacou-se como a mais frequente, foram notificados 564 casos de TB pulmonar, 38 casos de TB extrapulmonar e 4 casos em que as duas formas apresentaram-se em concomitância. Em relação ao tratamento da TB (Tabela 2), temos que 67,7\% dos casos evoluíram para a cura, $15 \%$ dos pacientes abandonaram o tratamento e $1,3 \%$ dos casos resultaram em óbito decorrente da TB.

Tabela 2 - Situação de encerramento dos casos confirmados de TB nos presídios paraibanos

\begin{tabular}{|l|l|}
\hline Situação de encerramento & Casos confirmados \\
\hline Ign/Branco & 8 \\
\hline Cura & 410 \\
\hline Abandono & 91 \\
\hline Óbito por tuberculose & 8 \\
\hline Óbito por outras causas & 9 \\
\hline Transferência & 80 \\
\hline Total & 606 \\
\hline
\end{tabular}

Fonte: Dados do Sistema de Informação de Agravos de Notificação - Ministério da Saúde (2018) 
De acordo com Valença et al. (2016), o abandono do tratamento entre os presidiários ocorre com maior frequência entre os presos mais jovens, com menor nível de escolaridade, com histórico de uso de álcool, com quadros redicivantes e que não receberam supervisão direta durante o tratamento.

\section{CONSIDERAÇÕES FINAIS}

Com base nos dados observados, percebe-se que o número de casos de tuberculose permanecem estáveis, tendo uma alta incidência nos presídios. Fatores como crescente taxa de ocupação prisional, sem a concomitante adequação de estrutura física e de recursos humanos, somada às condições precárias de higiene, ventilação e iluminação solar nas celas predispõem a riscos para o adoecimento de detentos e cria condições favoráveis à infecção e disseminação da TB (SOUZA, et al., 2012). Desse modo, é necessário instituir estratégias para diminuir tal incidência, melhorando a assistência à saúde e vida, com diagnóstico precoce e tratamento adequado; assim como, adequar a estrutura física, condições de ventilação e iluminação desses locais.

Percebeu-se que as prisões paraibanas corresponderam ao terceiro local com maior incidência de TB, com número maior de casos entre a população masculina, na faixa etária de 20-39 anos, pardos, com escolaridade da $1^{\mathrm{a}}$ a $4^{\mathrm{a}}$ série do ensino fundamental incompleta e com a forma pulmonar mais frequente. Com isso, deve-se investir em uma melhor estruturação das penitenciárias, a fim de sanar fatores de risco ergonômicos, como má ventilação e iluminação; principalmente nas penitenciárias masculinas, devido ao fato de a população carcerária constituir-se na maior parte por indivíduos desse sexo.

Além disso, deve-se investir na qualificação dos profissionais que lidam com a população privada de liberdade a fim de oferecer maior informação, e, também, na educação dos presos visto o fato de que a maior parte da população carcerária desconhece a respeito da infecção por tuberculose. Aliado a isso, é necessário haver diagnósticos precoces e tratamentos eficazes. Logo, conforme o próprio Ministério da Saúde, é necessário haver medidas efetivas provenientes da gestão pública, profissionais da saúde e sociedade para que se consiga mudar esse cenário que permanece por anos.

\section{REFERÊNCIAS BIBLIOGRÁFICAS}

ANDRZEYVSKI, A.; LIMBERGER, J.B. Tuberculose no Sistema Prisional: Revisão Sistemática da Epidemiologia, Diagnóstico e Tratamento Farmacológico. Disciplinarum Scientia. Série: Ciências da Saúde, Santa Maria, v. 14, n. 2, p. 189-198, 2013. Disponível em: http://sites.unifra.br/Portals/36/CSAUDE/2013-02/06\%20 (254).pdf. Acesso em: 19 jul. 2018. 
BRASIL. Ministério da Justiça. Departamento Penitenciário Nacional e Fórum Brasileiro de Segurança Pública. Infopen - Levantamento Nacional de Informações Penitenciárias. Brasília - DF. Disponível em: http://dados.mj.gov.br/dataset/ infopen-levantamento-nacional-de-informacoes-penitenciarias. Acesso em: 26 jul. 2018.

BRASIL. Ministério da Saúde. Secretaria Executiva. Datasus. Informações de Saúde. Epidemiológicas e Morbidade. Disponível em: http://www2.datasus.gov.br/ DATASUS/index.php?area=0203\&id=3 1009407. Acesso em 26 jul. 2018.

BRASIL. Ministério da Saúde. Secretaria de Vigilância em Saúde. Manual de recomendações para o controle da tuberculose no Brasil. 284p. Brasília, 2011. Disponível em: http://bvsms.saude.gov.br/bvs/publicacoes/manual_recomendacoes_ controle_tuberculose_brasil.pdf. Acesso em: 09 jul. 2018.

BRASIL. Ministério da Saúde. Secretaria de Vigilância em Saúde. Programa Nacional de Controle da Tuberculose. 2018. 111 slides. Disponível em: http:// portalarquivos2.saude.gov.br/images/pdf/2018/marco/19/APRES-PADRAO-JAN2018-REDUZIDA.pdf. Acesso em: 09 jul. 2018.

BRASIL. Ministério da Saúde. Secretaria de Vigilância em Saúde. Coordenação Geral de Desenvolvimento da Epidemiologia em Serviços. Guia de Vigilância em saúde: volume único [recurso eletrônico]. Brasília, 2017. Disponível em: http:// portalarquivos.saude.gov.br/images/pdf/2017/outubro/06/Volume-Unico-2017.pdf. Acesso em: 09 jul. 2018.

CONSELHO NACIONAL DE JUSTIÇA. Painel banco nacional de monitoramento de prisões - BNMP 2.0. Disponível em: https:// paineis.cnj.jus.br/qvajaxzfc/opendoc.htm?document=qvw_l\%2fpainelcnj. qvw\&host $=$ qvs\%40neodimioo3\&anonymous=true\&sheet=shbnmpiimapa. $\quad$ Acesso em: 21 out. 2018.

FORMIGA, N. S.; LIMA, D. A tuberculose em instituições prisionais: para além de uma epidemiologia, um estado de direito humano à saúde. Revista de Criminologia e Ciências Penitenciárias. v. 1, n. 2, 2011. Disponível em: http://www.procrim.org/ revista/index.php/COPEN/article/view/31. Acesso em: 26 jul. 2018.

LAROUZÉ, B. et al. Tuberculose nos presídios brasileiros: entre a responsabilização estatal e a dupla penalização dos detentos. Cad. saúde pública, Rio de janeiro, v. 31, n. 6, p. 1127-1130, jan. 2012. Disponível em: http://www.scielo.br/pdf/csp/ v31n6/0102-311 x-csp-31-6-1127.pdf.Acesso em: 21 out. 2018. 
MACHADO, C. et al. A incidência de tuberculose nos presídios brasileiros: revisão sistemática. Revista Brasileira Ciências da Saúde - São Caetano do Sul, v. 14, n. 47, p. 84-88, jan./mar., 2016. Disponível em: https://www.researchgate.net/ publication/312360723_A_incidencia_de_tuberculose_nos_presidios_brasileiros_ revisao_sistematica. Acesso em: 20 jul. 2018.

ORGANIZAÇÃO MUNDIAL DA SAÚDE (OMS). Prisons and health - 2014. Disponível em: http://www.euro.who.int/_data/assets/pdf_file/O005/249188/ Prisons-and-Health.pdf?ua=1. Acesso em: 09 jul. 2018.

PONCE, M.A.Z. et al. Atraso do diagnóstico da tuberculose em adultos em um município paulista em 2009: estudo transversal. Epidemiologia e Serviços de Saúde, Brasília, v. 25, n. 3, p. 553-562, set. 2016 . Disponível em http://scielo.iec. gov.br/scielo.php? script $=$ sci_arttext\&pid $=$ S 1679-49742016000300553\&lng=pt\&nr $\mathrm{m}=$ iso. Acesso em: 20 jul. 2018.

SÁ, A.M.M. et al. Causas de abandono do tratamento entre portadores de tuberculose. Revista Sociedade Brasileira de Clínica Médica. v. 15, n. 3, 2017. Disponível em: http://docs.bvsalud.org/biblioref/2017/11/875434/sbcm_153_155160.pdf. Acesso em: 27 out. 2018.

SACCHI, F.P.C. et al. Prisons as reservoir for community transmission of tuberculosis, Brazil. Emerging infectious disease, Atlanta, v. 21, n. 3, p. 452-455, mar. 2015. Disponível em: https://www.ncbi.nlm.nih.gov/pmc/articles/pmc4344267/. Acesso em: 09 jul. 2018.

SILVA, L.C.C. Pneumologia: Princípios e prática. Porto Alegre: Artmed, 2012. 1024 p.

SOARES, A.S et al. Prevalência da tuberculose pulmonar no sistema carcerário brasileiro. Revista COOPEX. V.6. p.1-10. 2015. Disponível em: https://www. researchgate.net/publication/286084343_Prevalencia_da_tuberculose_pulmonar_ no_sistema_carcerario_brasileiro. Acesso em: 26 jul. 2018.

SOUZA, K.M.J. et al. Atraso no diagnóstico da tuberculose em sistema prisional: a experiência do doente apenado. Texto contexto - enferm., Florianópolis , v. 21 , n. 1, p. 17-25, Mar. 2012. Disponível em: http://www.scielo.br/scielo.php?script=sci_ arttext\&pid=So104-07072012000100002\&lng=en\&nrm=iso. Acesso em 27 out. 2018.

SOUZA, K.M.J et al. Atraso no diagnóstico da tuberculose em sistema prisional: a experiência do doente apenado. Texto Contexto Enfermagem, Florianópolis, 21(1): 17-25; Jan-Mar, 2012

TAVARES, W.; MARINHO, L. A. C. Rotinas de diagnóstico e tratamento das doenças infecciosas e parasitárias. 4. ed. São Paulo: Atheneu, 2015. 
VALENÇA, M.S. et al. Tuberculose em presídios brasileiros: uma revisão integrativa da literatura. Ciência \& Saúde Coletiva, Rio de Janeiro, v. 21, n. 7, p. 2147-2 160, Jul 2016. Disponível em http://www.scielo.br/scielo.php? script=sci_ arttext\&pid=S141381232016000702 147\&lng=en\&nrm=iso. Acesso em: 08 Jul. 2018.

VITTI JUNIOR, W. O controle da tuberculose nos presídios: atuação das equipes de saúde na região (DRS VI) de Bauru/SP. Universidade Estadual Paulista, Faculdade de Medicina de Botucatu. Botucatu: [s.n], 2013. Disponível em: https://alsafi.ead. unesp.br/handle/1 1449/106048. Acesso em: 26 jul. 2018. 